\title{
Simultaneous Spectrophotometric Determination of Amitriptyline Hydrochloride and Chlordiazepoxide in Pharmaceutical Tablets by Multivariate Calibration Method
}

\author{
AMIR H.M. SARRAFI ${ }^{*}$, ZIBA KHODAKARAMI and MASOUMEH KARKEABADI \\ Chemistry Group, Faculty of Science, \\ Islamic Azad University (Central Tehran Branch), \\ P.O. Box 13185-768, Tehran, Iran, \\ a_mohsensarafi@iauctb.ac.ir
}

Received 10 March 2009; Accepted 4 April 2009

\begin{abstract}
The multivariate calibration method, partial least square regression (PLS) was applied for the simultaneous spectrophotometry determination of amitriptyline hydrochloride (AMT) and chlordiazepoxide (CHL) in their mixtures. The parameters of chemometric technique were optimized and the proposed method was validated with synthetic samples and applied to analyze these drugs in pharmaceutical products with good accuracy and precision. The results were compared with those given by United State Pharmacopoeia method. The square of the correlation coefficients $\left(\mathrm{R}^{2}\right)$ for predicted AMT and CHL with the proposed method in a test sample were 0.9951 and 0.9897 respectively. The relative standard deviation for commercial tablets in the proposed method and USP standard method were 0.42 and $2.61 \%$, respectively.
\end{abstract}

Keywords: Amitriptyline hydrochloride, Chlordiazepoxide, Pharmaceutical analysis, Spectrophotometric, Multivariate calibration.

\section{Introduction}

Amitriptyline hydrochloride 10,11 -dihydro- $N, N$-dimethyl-5H-dibenzo $[a, d]$ cycloheptane- $\Delta 5 \gamma$ propylamine hydrochloride (AMT) is used commonly in combination with chlordiazepoxide 7chloro-2-(methyl-amino)-5-phenyl-3H-1,4-benzodiazepine-4-oxide (CHL). The mixture of these two drugs is used for the treatment of patients with moderate to severe depression associated with moderate to severe anxiety. Therefore, the determination of these drugs is a frequent analytical problem in quality control of the pharmaceutical industries. The two drugs studied in this work show a strong overlap between their absorption spectra. 
Hence their simultaneous determination is hard when conventional spectrophotometric techniques are used $1^{-3}$. Normally the method used to resolve a complex mixture of these drugs is mainly HPLC ${ }^{4}$.

In the recent years, multivariate calibration methods have been used to resolve mixtures of two or more compounds with similar spectral characteristics. Partial Least Squares (PLS) is a multivariate calibration method based on factor analysis. The basic concept of PLS regression was originally developed by $\mathrm{Wold}^{6}$, and the use of the PLS method for chemical analysis was pioneered by Wold et $a l^{6,7}$. A detailed description on the mathematical principles of the PLS algorithms have been reported by Martens et al. ${ }^{8}$ and others ${ }^{9-11}$.

In most cases, multivariate methods for evaluation of spectroscopic data have advantages as simplicity and cheapness. Therefore, these methods have been applied to the determination of drugs ${ }^{12-16}$, because of HPLC methods and conventional spectroscopic methods were slow, expensive and complex.

The aim of this paper is to investigate the ability of PLS and PCR methods for quantifying binary mixtures of amitriptyline hydrochloride and chlordiazepoxide without prior separation and to apply the optimized method in pharmaceutical preparations.

\section{Experimental}

Commercial samples were bought from pharmacies. Analytical grade AMT and CHL were obtained from Food and Drug Control Laboratories (Tehran, Iran). All other chemical and solvents were of analytical reagent grade.

\section{Apparatus and software}

A Jasco 530 scanning spectrophotometer connected to PC fitted data software and Agilent 1200 HPLC were used for all the measurements.

The Chemometrics toolbox and Statistic toolbox of Matlab 7.1 software was used for the statistical treatment of the data and application of various multivariate methods.

\section{Procedure}

Accurately weighed amounts of finely powdered pure ATM and CHL were placed in two $50 \mathrm{~mL}$ volumetric flasks and methanol was added. The volumetric flasks were subjected to ultrasonication for 15 minutes by a laboratory ultrasonic water bath. After cooling and further dilution with methanol, the calibration and synthetic mixtures mixing of these two solutions with different ratios produced test sets of samples. After grinding and homogenizing of an accurately weighed of 20 tablets, $0.2787 \mathrm{~g}$ of sample was used for analysis. Each weighed sample was mixed with $40 \mathrm{~mL}$ methanol and the mixture was subjected to ultrasonication for 15 minutes. After cooling and further dilution to $50 \mathrm{~mL}$ with methanol, a $10 \mathrm{~mL}$ portion of the sample was centrifuged at $3000 \mathrm{rev} / \mathrm{min} .1 \mathrm{~mL}$ portion of the supernatant was then diluted to $100 \mathrm{~mL}$ with methanol. The absorption spectra between 200 and $400 \mathrm{~nm}$ against methanol were recorded for all solutions. Commercial tablets were also analyzed, using United States Pharmacopoeia (USP) method ${ }^{4}$.

\section{Results and Discussion}

\section{$U V$-Vis spectra of AMT and CHL}

The absorption spectra of standard AMT and CHL solutions recorded between 200 and 400 $\mathrm{nm}$ are shown in Figure 1. The two drugs studied show a strong overlap in their absorption spectra and univariate analysis method can not be applied for resolving this mixture. 


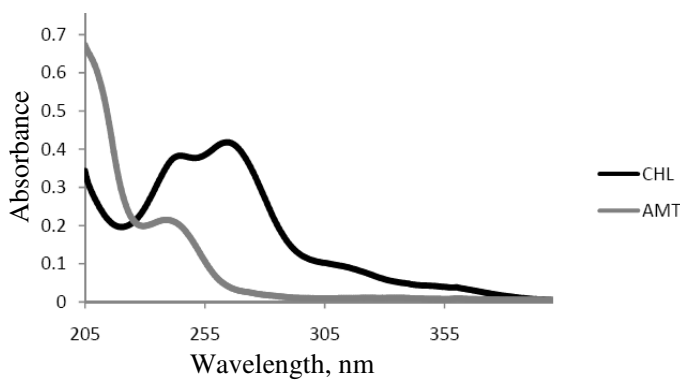

Figure 1. The UV-Vis absorption spectra AMT and CHL standard solutions.

\section{Experimental design of sample sets}

Calibration and test sets for two component systems were designed according to factorial principles. Solutions containing drug concentrations in the range 0.0-10.0 ppm for AMT and 0.0-4.0 ppm for CHL were produced by dilution of the stock solutions. A five level factorial design was used to produce a full set of 25 samples. A three level set was derived from this to produce a calibration set of nine samples, with the remaining 16 samples used for an independent test set ${ }^{17}$. The compositions of the used calibration and test sets are summarized in Table 1 and Table 2 respectively.

Table 1. Calibration set composition.

\begin{tabular}{ccc}
\hline Standard & AMT,mg/L & CHL,mg/L \\
\hline P1 & 0.0 & 0.0 \\
P2 & 0.0 & 2.0 \\
P3 & 0.0 & 4.0 \\
P4 & 5.0 & 0.0 \\
P5 & 5.0 & 2.0 \\
P6 & 5.0 & 4.0 \\
P7 & 10.0 & 0.0 \\
P8 & 10.0 & 2.0 \\
P9 & 10.0 & 4.0 \\
\hline
\end{tabular}

Table 2. Test set composition.

\begin{tabular}{ccc}
\hline Sample & AMT, mg/L & CHL, mg/L \\
\hline S1 & 0.0 & 1.0 \\
S2 & 0.0 & 3.0 \\
S3 & 2.5 & 0.0 \\
S4 & 2.5 & 1.0 \\
S5 & 2.5 & 2.0 \\
S6 & 2.5 & 3.0 \\
S7 & 2.5 & 4.0 \\
S8 & 5.0 & 1.0 \\
S9 & 5.0 & 3.0 \\
S10 & 7.5 & 0.0 \\
S11 & 7.5 & 1.0 \\
S12 & 7.5 & 2.0 \\
S13 & 7.5 & 3.0 \\
S14 & 7.5 & 4.0 \\
S15 & 10.0 & 1.0 \\
S16 & 10.0 & 3.0 \\
\hline
\end{tabular}




\section{Selection of the optimum number of factors and the spectral region}

To select the correct number of factors in the PLS algorithm a cross validation method, leaving out one sample at a time, was employed ${ }^{8}$.

For the set of 25 spectra, PLS-2 calibration on 24 calibration spectra were performed and using this calibration, the concentration of the samples left out during the calibration process was performed. These processes were repeated a total of 25 times until each sample had been left out once. The predicted concentrations $\left(\mathrm{X}_{\mathrm{ij}}^{\wedge}(\mathrm{k})\right)$ of the compounds in each sample obtained with $\mathrm{k}$ factors, were compared with the already known concentrations $\left(\mathrm{X}_{\mathrm{ij}}\right)$ and the root mean square error of cross-validation (RMSECV) was calculated for each of the $\mathrm{k}$ factor levels as follows:

$$
\operatorname{RMSECV}(\mathrm{k})=\left(\sum_{i=1}^{r} \sum_{j=1}^{c}\left(X_{i j}-X_{i j}^{\wedge}(k)\right)^{2}\right)^{1 / 2}
$$

The optional value for $\mathrm{k}$ is the level that yields the smallest RMSECV $(\mathrm{k})^{8}$. To check the selected factor number, the external validation method was also used by simply computing root mean squared error of predication (RMSEP) for I objects in test set for each of the $\mathrm{k}$ factor levels as follows:

$$
\operatorname{RMSEP}(\mathrm{k})=\left(\sum_{i=1}^{r} \sum_{j=1}^{m}\left(Z_{i j}-Z_{i j}^{\wedge}(k)\right)^{2} / I\right)^{1 / 2}
$$

Where, $\mathrm{Z}_{\mathrm{ij}}$ is known concentrations in test set and $\mathrm{Z}_{\mathrm{ij}}(\mathrm{k})$ is predicted concentrations with $\mathrm{k}$ factors ${ }^{8}$. This showed that the external test set validation and the internal cross validation indicated about the same number of factors.

To select the spectral region, all of the top steps used repeatedly and the spectral region that leading to the lowest values of RMSEP was selected ${ }^{12}$. The spectral region between 205 and $305 \mathrm{~nm}$ was selected for analysis and as a consequence 101 wavelengths were used.

The optimal number of factors and RMSEP and RMSECV values obtained by

PLS-2 algorithm is summarized in Table 3.

Table 3. RMSEP and RMSECV values.

\begin{tabular}{ccccc}
\hline & & Factors & RMSEP & RMSECV \\
\hline PLS-2 & AMT & 2 & 0.226502 & 0.222684 \\
& CHL & 2 & 0.163356 & 0.137266 \\
\hline
\end{tabular}

The proposed PLS-2 calibration model was evaluated by prediction of drugs concentrations in their own designed calibration set, obtaining recoveries between 94.68$101.26 \%$ for AMT and $96.6-101.88 \%$ for CHL.

\section{Statistical parameters for the optimized model}

Using the internal validation in their own designed calibration set, the following statistical parameters have been obtained:

a) The values of root mean square error of calibration (RMSEC), which is an indication of the average error in the analysis for each component.

b) The square of the correlation coefficients $\left(\mathrm{R}^{2}\right)$, which is an indication of the quality of the straight line that fits the data.

In the Table 4, the results obtained for these parameters by PLS-2 are shown. We can see that the $\mathrm{R}^{2}$ values are in all cases very near to 1 which is an indication of similarity between predicted and known values. On the other hand, in general terms, the statistical parameters obtained by PLS-2 are good. 
Table 4. Statistical parameters of the model optimized.

\begin{tabular}{lcc}
\hline & \multicolumn{2}{c}{ PLS-2 } \\
\cline { 2 - 3 } & RMSEC & $\mathrm{R}^{2}$ \\
\hline AMT & 0.153410 & 0.9986 \\
CHL & 0.062310 & 0.9985 \\
\hline
\end{tabular}

\section{External validation of PLS-2 calibration model}

16 synthetic mixtures in test set were predicted by applying PLS-2 methods. The square of correlation coefficients $\left(\mathrm{R}^{2}\right)$ and recovery range for these synthetic solutions are summarized in Table 5. Satisfactory values are obtained in most of mixtures analyzed by the methods.

Table 5. Recovery range and $\mathrm{R}^{2}$ for synthetic mixtures in test set.

\begin{tabular}{ccccc}
\hline \multicolumn{2}{c}{ AMT } & \multicolumn{2}{c}{ CHL } \\
\hline PLS2 & \%Rec. range & $\mathrm{R}^{2}$ & \%Rec. range & $\mathrm{R}^{2}$ \\
\cline { 2 - 5 } & $96.36-102.33$ & 0.9951 & $95.7-105.17$ & 0.9897 \\
\hline
\end{tabular}

\section{Analysis of commercial samples}

Commercial tablets were analyzed by using two methods, the proposed spectrophotometric method and USP standard method ${ }^{4}$. Results are summarized in Table 6. As can be seen satisfactory results were obtained in all cases by the proposed methods. PLS-2 results appear similar for AMT and CHL to USP results.

Table 6. Analysis of commercial tablets.

\begin{tabular}{lcc}
\hline & AMT $\pm \%$ RSD & CHL $\pm \%$ RSD \\
\cline { 2 - 3 } Declared Contents & 25 & 10 \\
PLS-2 Results & $25.68 \pm 0.42$ & $10.43 \pm 0.24$ \\
USP Results & $25.55 \pm 3.65$ & $10.22 \pm 2.61$ \\
\hline
\end{tabular}

Statistical parameters obtained by replicate analysis for the commercial tablets by proposed PLS-2 and USP standard methods are also given in Table 6. These results show that the precision of the proposed method is better than USP method. Excipients frequently added to dosage forms did not interfere with the proposed method.

\section{Conclusion}

A comparative study of the use of PLS-2 for the resolution and simultaneous determination of AMT and CHL in a binary mixture has been accomplished, showing that this method provide a clear example of the high resolving power of these techniques. In several terms, similar results were obtained for these two drugs in both synthetic and commercial applications by PLS-2.

The results obtained confirm the suitability of the proposed method for accurate and precise analysis of Amitriptyline Hydrochloride and Chlordiazepoxide in pharmaceutical preparations, since they were applied directly to the commercial preparations without previous treatment. Also no expensive (dissolution and injection) laboratory technique is needed. In addition the proposed methods are suitable for application without interference of the excipients.

\section{References}

1. Abuirjeie M A and Abdel-Hamid M E, Anal Lett., 1989, 22(4), 951-962.

2. Sane R T, Gangal D P, Tendolkar R V, Kothurkar R M and Ladage K D, Indian J Pharm Sci., 1989, 51(2). 
3. Ozkan S A, Erk N and Senturk Z, Anal Lett.,1999, 32(3), 497-520.

4. United States pharmacopeia convention, INC. Rand Menally, Taunton, M A, 1995.

5. Wold H, in Research Papers in Statistics, (David F, Eds), Wiley, New York, 1966.

6. Wold H, in Systems under Indirect Observations, (H. Joreskog and H. Wold, Eds), North-Holland, Amesterdam, 1982.

7. Wold S, Martens $\mathrm{H}$ and Wold $\mathrm{H}$, in The Multivariate Calibration Problem in Chemistry solved by PLS, Matrix Pencils (Lecture Notes in Mathematics), (A. Ruhe and Kagstrom B, Eds), Springer, Heidelberg, 1992.

8. Martens H and Naes T, Multivariate Calibration, Wiley, Chichester, 1992.

9. Long J R and Gregori V G, Anal Chem., 1991, 62, 1791.

10. Bos M, Bos A and Van der Linden W E, Analyst, 1993, 118, 323.

11. Despagne F and Massart D L, Analyst, 1998, 123, 157A.

12. Kargosha K and Sarrafi A H M, J Pharm Biomed Anal., 2001, 26, 273-279.

13. Ferraro M C F, Castellano P M and Kaufman T S, J Pharm Biomed Anal., 2004, 34, 305-314.

14. Markopoulou C K, Malliou E T and Koundourellis J E, J Pharm Biomed Anal., 2005, 37, 249-258.

15. Hadad G M, El-Gindy A and Mahmoud W M M, Spectrochim Acta Part A, 2008, 70, 655-663.

16. Khoshayand M R, Abdollahi H, Shariatpanahi M, Saadatfard A and Mohammadi A, Spectrochim Acta Part A, 2008,70,491-499,

17. Bayne C K, Rubin I B, Experimental Design, VCH, New York, 1986. 


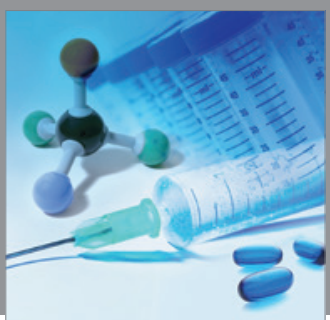

International Journal of

Medicinal Chemistry

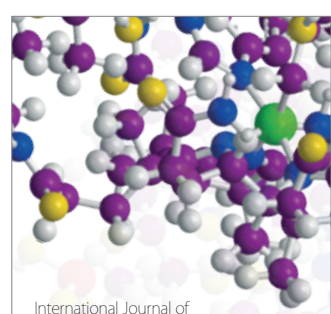

Carbohydrate Chemistry

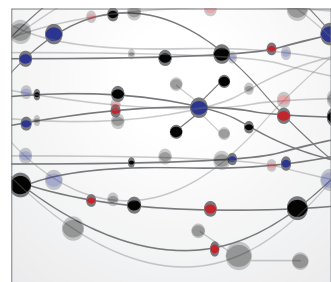

The Scientific World Journal
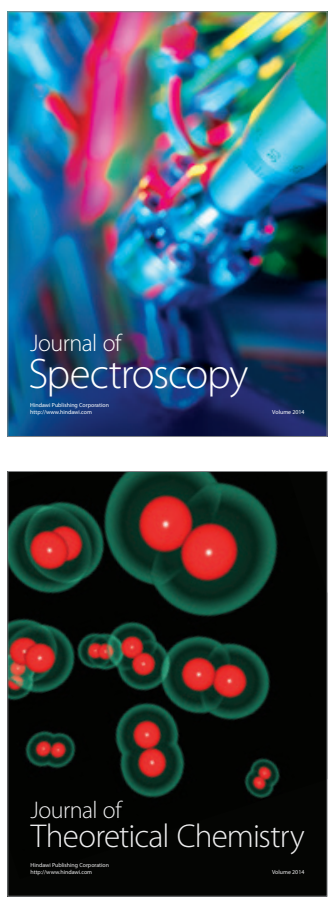
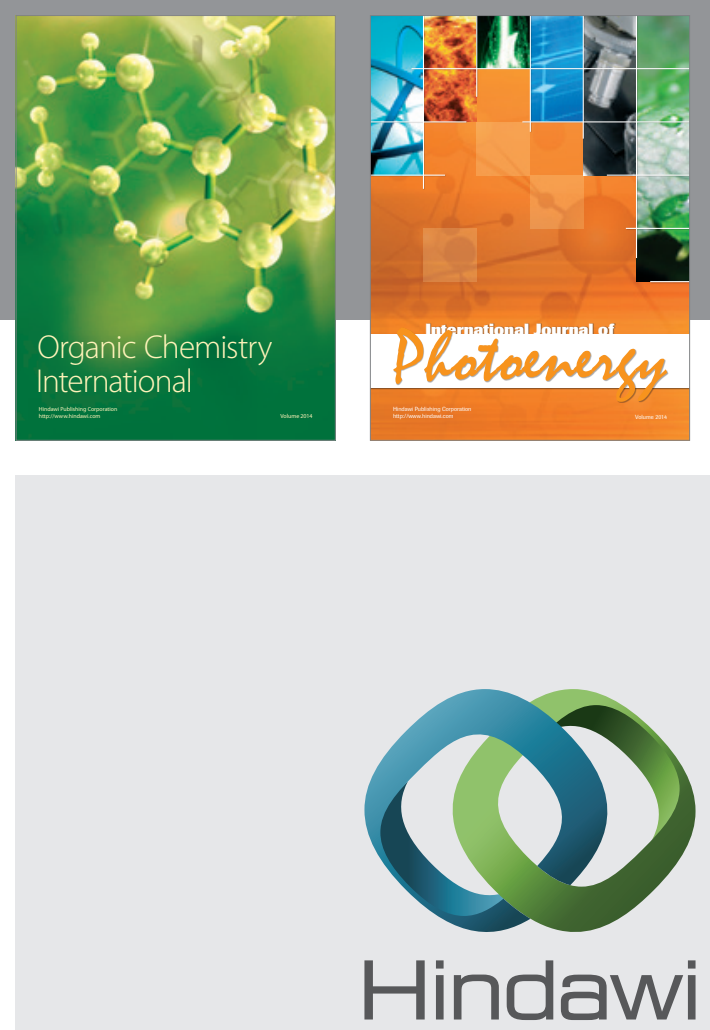

Submit your manuscripts at

http://www.hindawi.com
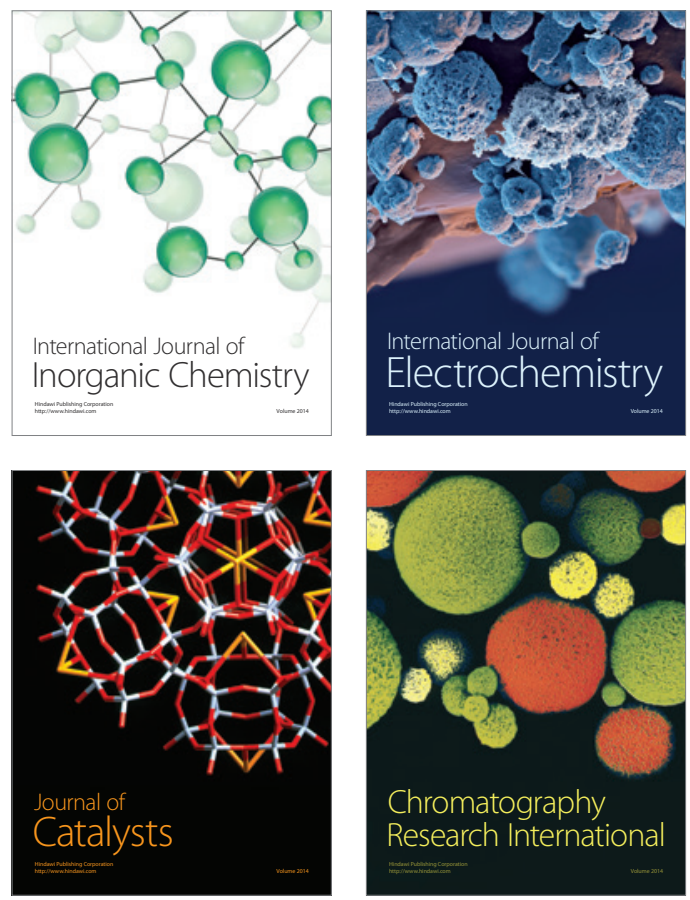
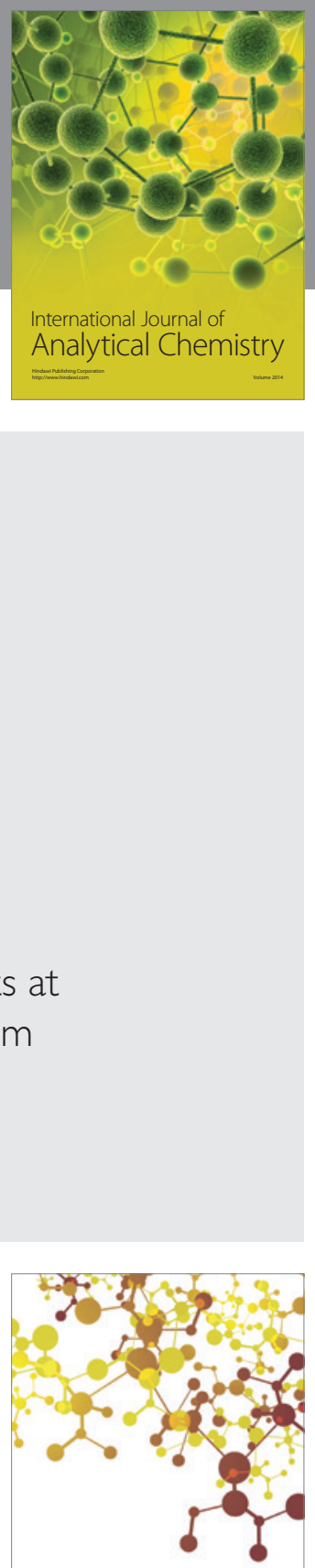

Journal of

Applied Chemistry
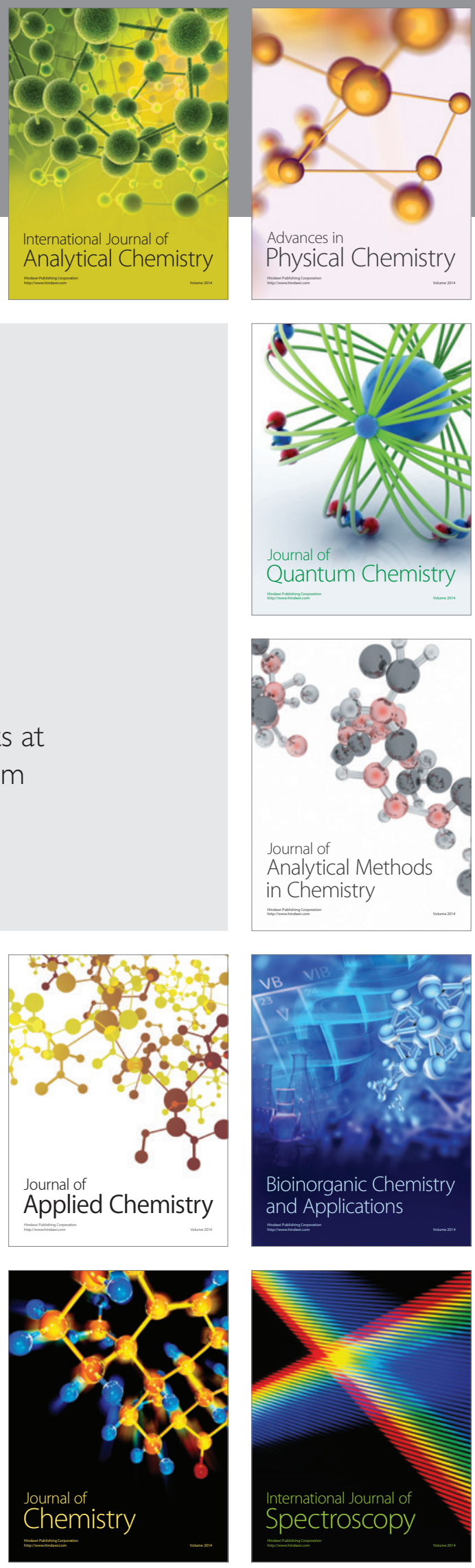